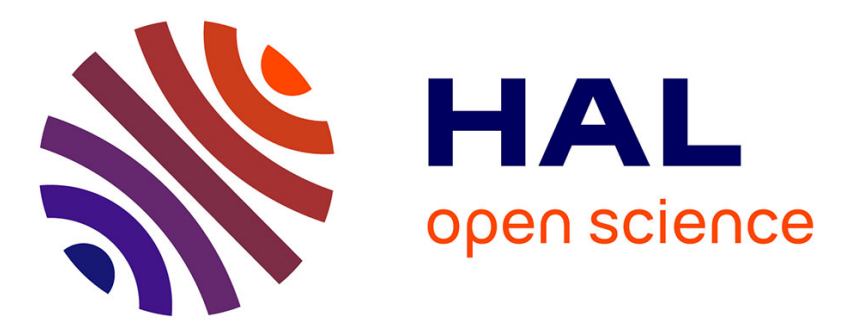

\title{
Covalent organic frameworks from a monomer with reduced symmetry: polymorphism and Sierpiński triangles
}

Daling Cui, Yuan Fang, Oliver Maclean, Dmitrii F. Perepichka, Federico Rosei, Sylvain Clair

\section{To cite this version:}

Daling Cui, Yuan Fang, Oliver Maclean, Dmitrii F. Perepichka, Federico Rosei, et al.. Covalent organic frameworks from a monomer with reduced symmetry: polymorphism and Sierpiński triangles. Chemical Communications, 2019, 90, pp.13586-13589. 10.1039/C9CC05674B . hal-02355234

\section{HAL Id: hal-02355234 \\ https://hal-amu.archives-ouvertes.fr/hal-02355234}

Submitted on 8 Nov 2019

HAL is a multi-disciplinary open access archive for the deposit and dissemination of scientific research documents, whether they are published or not. The documents may come from teaching and research institutions in France or abroad, or from public or private research centers.
L'archive ouverte pluridisciplinaire HAL, est destinée au dépôt et à la diffusion de documents scientifiques de niveau recherche, publiés ou non, émanant des établissements d'enseignement et de recherche français ou étrangers, des laboratoires publics ou privés. 


\title{
Covalent organic frameworks from a monomer with reduced symmetry: polymorphism and Sierpiński triangles
}

\author{
Daling Cui, ${ }^{a,+}$ Yuan Fang, ${ }^{b,+}$ Oliver MacLean, ${ }^{a}$ Dmitrii F. Perepichka*,b, Federico Rosei*,a ${ }^{*}$ Sylvain \\ Clair $*, a, c$
}

\begin{abstract}
We report on the synthesis of a covalent organic framework based on the low-symmetry 1,3-benzenediboronic acid precursor. Two distinct polymorphs are obtained, a honeycomb network and Sierpiński triangles, as elucidated by scanning tunneling microscopy. Control on the polymorph formation was achieved by varying the precursor concentration for on-surface synthesis.
\end{abstract}

Covalent organic frameworks (COFs), pioneered by Yaghi and co-workers in $2005,{ }^{1}$ are porous crystalline organic polymers with great potential for several applications. ${ }^{2-4}$ The crystallinity in COFs is achieved through the proper design of the building blocks which assemble in periodic structures as the thermodynamic minima and the use of dynamic (reversible) covalent bonds which prevent kinetically locked defects (mislinks) in these solids. ${ }^{2}, 5$ Typically, two-dimensional (2D) COFs are built from monomers with $\mathrm{C}_{2}, \mathrm{C}_{3}$ or $\mathrm{C}_{4}$ symmetry so that the generated networks can easily fulfill the geometric requirement imposed by $2 \mathrm{D}$ confinement, 2,5 and a lowsymmetry building unit would tend to produce amorphous structures. ${ }^{6}$ The use of low-symmetry linkers in metal-organic frameworks (MOFs) is very common but still remains underdeveloped in COFs. ${ }^{7-9}$ This could however be particularly useful to expand the COF family, to include complex tessellation patterns, and to create multiporous systems, ${ }^{10}$ similar to the case of MOFs. ${ }^{11,} 12$

In the on-surface synthesis approach, ${ }^{13}, 14$ single sheets of lamellar COFs or 2D covalent networks can be formed on a substrate and characterized by scanning tunneling microscopy (STM). Single sheets of COF-1, ${ }^{1}$ otherboroxine COFs ${ }^{15-21}$ as well

\footnotetext{
a. Centre Énergie, Matériaux et Télécommunications, Institut National de la Recherche Scientifique, 1650 Boulevard Lionel-Boulet, Varennes, Québec J3X 1S2, Canada.

b. Department of Chemistry, McGill University, 801 Sherbrooke Street West, Montreal, Québec H3A OB8, Canada.

Aix Marseille Univ, Université de Toulon, CNRS, IM2NP, Marseille, France. Author Contributions

† D.C. and Y.F. contributed equally.

* Corresponding authors: dmitrii.perepichka@mcgill.ca, rosei@emt.inrs.ca sylvain.clair@univ-amu.fr

Electronic Supplementary Information (ESI) available: [Experimental details and additional experimental data (IR, NMR, BET, STM images)].
}

as imine-linked (Schiff-base) COFs. ${ }^{22,} 23$ have been successfully synthesized on solid surfaces. High-symmetry precursors are mainly used, and networks synthesized using low-symmetry monomers have been rarely reported on surfaces. ${ }^{15,24,25}$

Sierpiński triangle (ST) is a fractal geometrical set with an equilateral triangular shape subdivided recursively into smaller equilateral triangles. Because it possesses a reduced Hausdorff dimension of 1.58 , such a pattern is attractive to build nanoscale structures with unique properties. ${ }^{26,27}$ In principle, the combination of three-fold and $120^{\circ} \mathrm{V}$-shaped nodes is the basic requirement for creating Sierpiński triangle selfassembly. ${ }^{28-30}$ Molecular based Sierpiński triangles have been experimentally achieved on single-crystalline surfaces ${ }^{31}$ via supramolecular structures such as halogen bonding, ${ }^{29}$ hydrogen bonding, ${ }^{32}$ and metal-organic coordination ${ }^{33-37}$ or in the form of covalently-bonded polymers. ${ }^{30,} 38,39$

To grow a COF from a low-symmetry monomer, we selected 1,3benzene diboronic acid (1,3-BDBA) with a reduced $\boldsymbol{D} \mathbf{1}$ symmetry as the monomer (Fig. 1). 40 This V-shaped ditopic linker can selfcondense into a 3 -fold node with a boroxine ring through a dehydration reaction. Depending on the rotational configuration around the boroxine ring, two kinds of elementary nodes can form based on a homotactic or a heterotactic motif (Fig. 1), and these give rise to two distinct polymorphs. In the homotactic motif, the three phenyl 'arms' are related by a threefold rotational axis of symmetry forming a windmill structure. This represents the repeating unit for an extended honeycomb 2D COF with each unit cell including 3 benzene moities. On the other hand, the heterotactic motif has no symmetry (Fig. 1). The heterotactic node is the basic structural unit for building Sierpiński triangles. While this article was in preparation, the successful realization of STs from 1,3BDBA precursors was communicated by Mo and coworkers, 39 but no observation of extended 2D honeycomb COF has been reported so far. 
Here, we report on the synthesis of bulk microcrystalline COF from the low-symmetry precursor 1,3-BDBA and on-surface growth of the 2D honeycomb COF and ST polymorphs (Fig. 1). Bulk microcrystalline COF was obtained by the solvothermal method, as confirmed by infrared (IR) and solid-state NMR (see Figs. S1, S2). The 1,3-BDBA COF exhibited low crystallinity, indicated by diffraction peaks in powder $X$-ray diffraction (PXRD) data (Fig. 2a), though relatively broadened as compared to other boronic acid COFs constructed from high symmetry 1,4BDBA monomer. ${ }^{1}$ Full width at half maximum (FWHM) for 110 reflection is $\sim 2.8^{\circ}$, which corresponds to a correlation length of $\sim 3 \mathrm{~nm}$ in the lateral (a-b) direction. The FWHM for 001 reflection is $\sim 1.7^{\circ}$ and corresponds to a correlation length of $\sim 5$ $\mathrm{nm}$ in the stacking (c) direction. PXRD simulations were carried out to elucidate the packing motif of the COF. Rather than the more common eclipsed stacking, the staggered stacking was in better agreement with the experimental result in terms of peak positions and relative intensities (Fig. 2 and Fig. S3). The simulated cell parameters are $a=b=13.5 \AA, c=9.1 \AA \AA \alpha=60.0^{\circ}$, $\beta=59.5^{\circ}, \gamma=115.0^{\circ}$. The stacking along the $c$-axis has an interlayer distance of $3.4 \AA$.

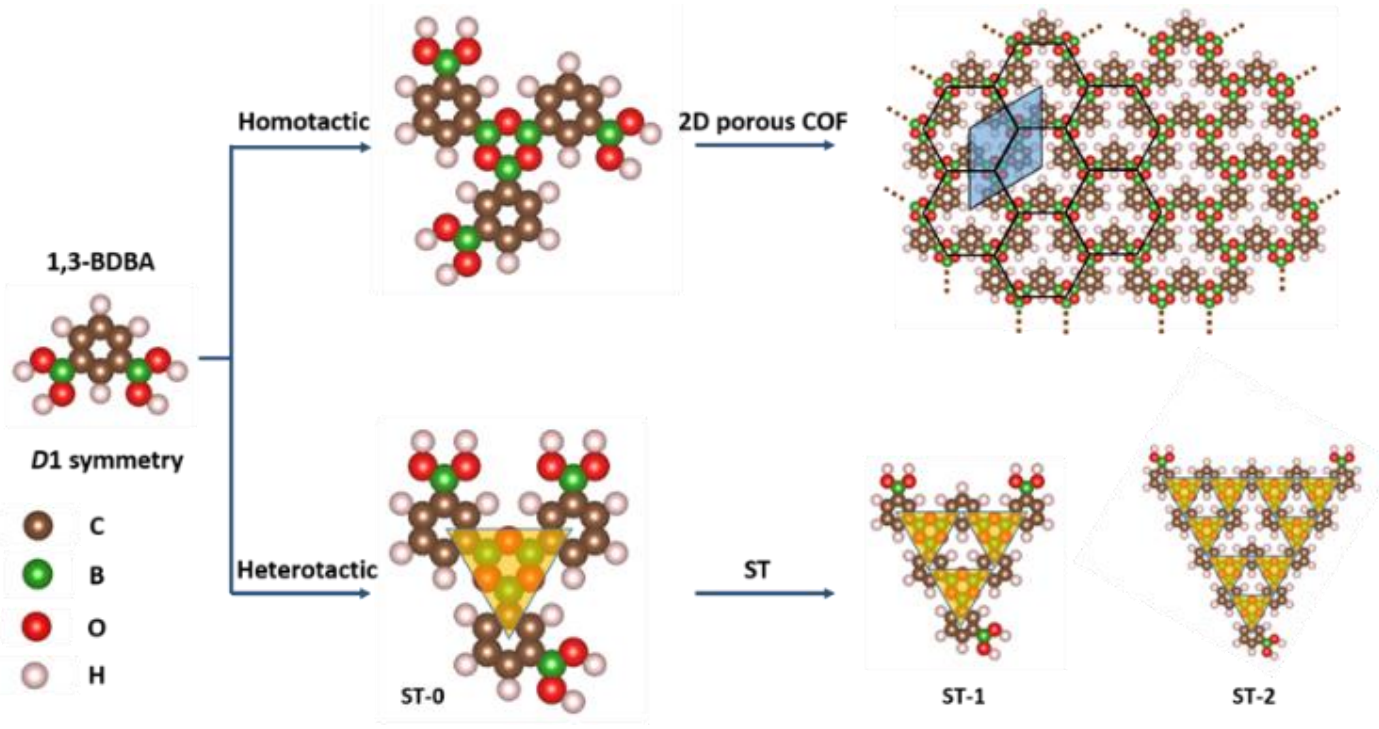

Fig. 1 Formation of homotactic 2D COF and heterotactic clusters (Sierpiński triangles, ST) by polycondensation of 1,3-BDBA. The unit cell of the 2D COF (honeycomb Fig. 1 Formation of homotactic $2 \mathrm{D}$ COF and heterotactic clusters (Sierpiński triangles, ST) by polycondensation of 1,3-BDBA. The unit cell of the 2D COF (hile
network) is indicated by a shaded blue rhombus. In the STs, the superimposed yellow shaded triangles help visualize the order ( $n$ ) of the triangles (ST- $n$ ).

1,3-BDBA COF has a higher-density packing structure compared to 1,4-BDBA COF and it is nearly pore-less (Fig. 2b). The van der Waals diameter of the pores is only $1 \AA$, i.e. too small for any gas molecule. Accordingly, the $\mathrm{N}_{2}$ adsorption analysis shows a low Brunauer-Emmett-Teller (BET) surface area of $49 \mathrm{~m}^{2} / \mathrm{g}$ (Fig. S4), which can be attributed to adsorption on the surface and in lattice defects.

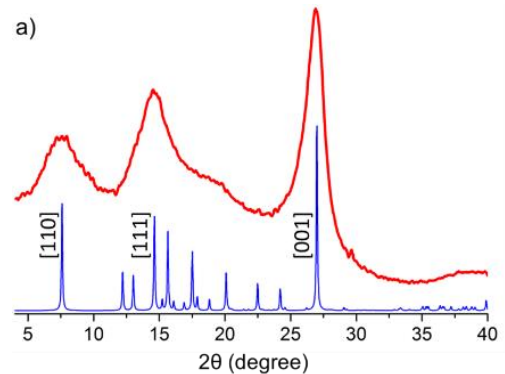

b)

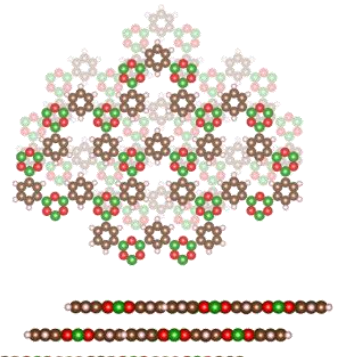

Fig. 2. PXRD patterns and simulated crystal structure of 1,3-BDBA COF. a) Experimental PXRD of as-synthesized COF (red lines) and simulated XRD pattern (blue lines) using the staggered arrangement 1,3-BDBA. b) Top and side views of the simulated $\mathrm{A}, c=9.1 \AA$; $\alpha=60.0^{\circ}, \beta=59.5^{\circ}, \gamma=115.0^{\circ}$.

The relatively low crystallinity of the 1,3-BDBA COF may be explained by the presence of heterotactic nodes while the ideal COF is based on homotactic nodes. In particular, ST structures are aperiodic and possess only 3 active bonding sites, one at each of the triangle corners (Fig. 1). Their accommodation in different domains is therefore limited and their presence is probably an important source of disorder.

To gain insight into the competition between the honeycomb and ST structures, we performed on-surface growth of the covalent networks. On highly oriented pyrolytic graphite (HOPG), extended ordered networks formed in the highcoverage regime, as identified by STM. Two distinct polymorphs are unambiguously observed (Fig. 3). For boronic acid based COFs, only the benzene rings are visible as bright protrusions and the boroxine rings are not observed by STM. ${ }^{15-17, ~ 19, ~ 20, ~} 41$ In Fig. 3a, the periodic arrangement of the benzenes in a hexagonal lattice makes it impossible to discriminate between the empty pores and the boroxine rings. Therefore the real unit cell of this honeycomb-like network cannot be determined from the STM image alone. However, the homogeneous appearance and the periodicity of the image are consistent with the honeycomb network formed by homotactic nodes. 


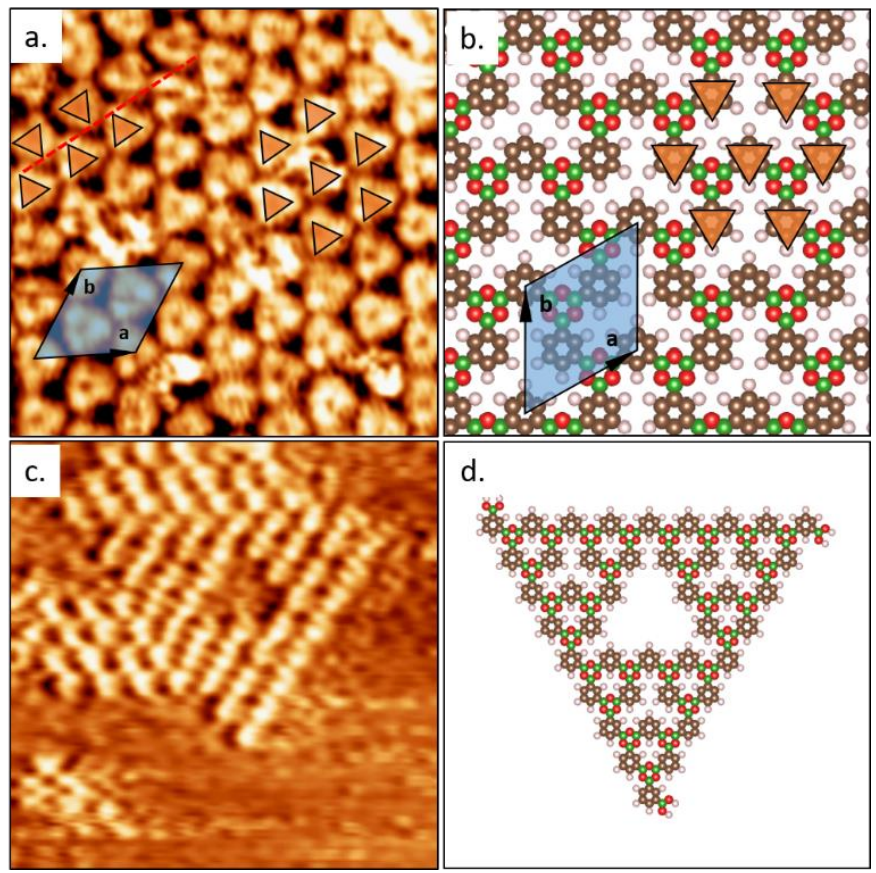

Fig. 3. a) STM image of extended honeycomb-like 2D COFs, collected at the heptanoic acid/HOPG interface. Tunnelling conditions: $V_{\text {bias }}=-1500 \mathrm{mV}, I_{\text {set }}=50$ pA. Image dimension: $6.0 \times 6.0 \mathrm{~nm}^{2}$. The orange triangles indicate the positions of effective honeycomb network is indicated $(a=b=1.30 \pm 0.13 \mathrm{~nm}, \alpha=60.3 \pm 5.9)$. b) Corresponding schematic representation of the network. c) STM image of a ST3 pattern. Tunnelling conditions: $V_{\text {bias }}=-1200 \mathrm{mV}$, $I_{\text {set }}=50 \mathrm{pA}$. Image dimension $10.0 \times 10.0 \mathrm{~nm}^{2}$. d) Schematic representation of ST-3.

The honeycomb network formed with 1,3-BDBA has p31m symmetry, in contrast to the honeycomb network with higher p6mm symmetry obtained from 1,4-BDBA. This implies the existence of two equivalent mirror-symmetric domains, which can be distinguished due to the apparent triangular shape of the individual benzene rings with two mirror symmetric
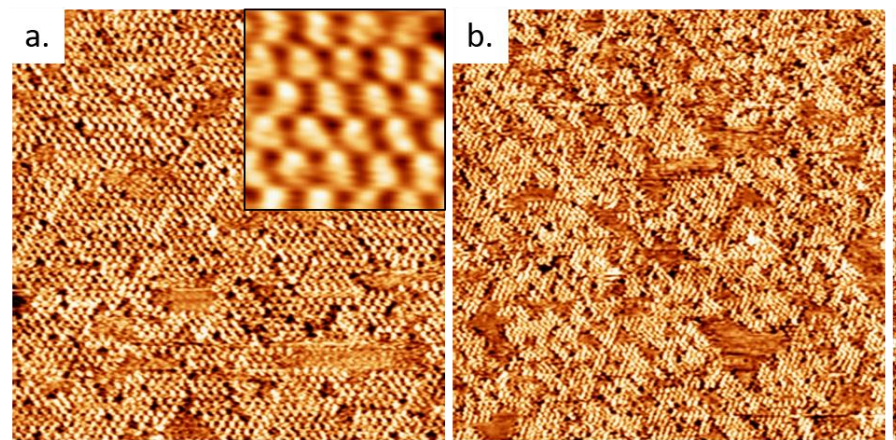

Fig. 4. Effect of 1,3-BDBA concentration on the network morphology. STM images collected at the heptanoic acid/HOPG interface of the sample prepared with a concentration of a) $1.2 \times 10^{-3} \mathrm{M}$, b) $\left.0.6 \times 10^{-3} \mathrm{M}, \mathrm{c}\right) 0.3 \times 10^{-3} \mathrm{M}$. d) Zoomed-in images extracted from (c) with corresponding molecular models. Image dimensions: a) 40 $\times 40 \mathrm{~nm}^{2}$
$=50 \mathrm{pA}$.

High-concentration conditions favor the formation of $2 D$ honeycomb-like network while low-concentration conditions favor the ST polymorph. The precursor concentration has been previously shown as the relevant parameter to control the formation of different polymorphs. ${ }^{14,42}$ In ultrahigh vacuum conditions similar coverage-dependent polymorphism has been identified in the supramolecular ST systems. ${ }^{29,} 32,43$ For example, the V-shaped molecule 4,4"-dibromo-1,1':3',1"-terphenyl $(\mathrm{B} 3 \mathrm{~Pb})$ can form honeycomb-like networks and ST structures depending on the molecular coverage on $\mathrm{Ag}(111) .{ }^{29}$
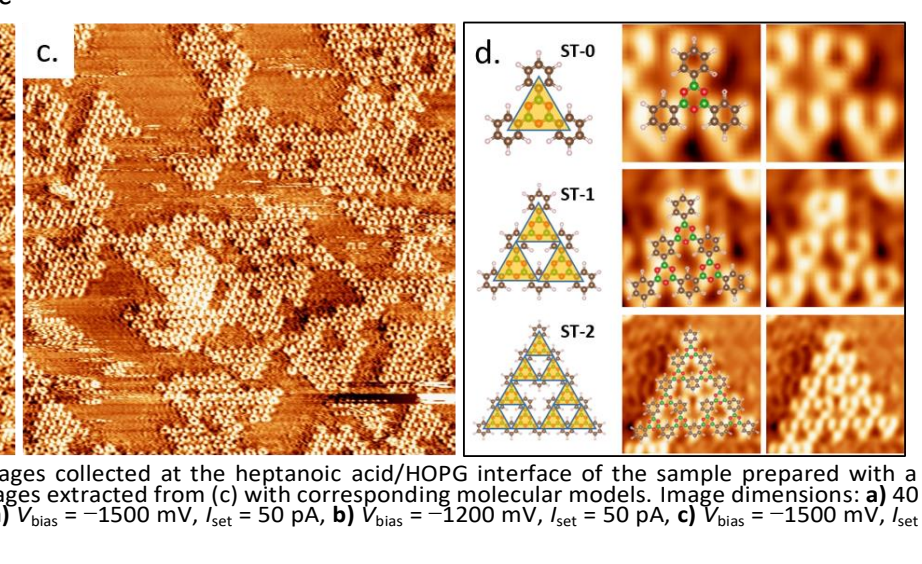

orientations. The domain boundaries appear mostly linear (top of Fig. 3a and Fig. S5). The apparent arrangement of the benzene rings in a hexagonal lattice is exactly preserved across the domain boundaries and extends seamlessly over several orientational domains (Fig. S6), despite a completely different bonding configuration (non-covalent) along the domain boundary.

The formed polymorphs can be controlled by the concentration of the monomer solution. The STM images shown in Fig. 4 were collected from the samples prepared with different concentrations of 1,3-BDBA solution. With a precursor concentration of $1.2 \times 10^{-3} \mathrm{M}$, the self-condensation of $1,3-\mathrm{BDBA}$ molecules forms high-density honeycomb-like 2D COFs which nearly cover the entire surface (Fig. 4a). The overall quality of the monolayer is similar to that of COF-1 synthesized by the same procedure. ${ }^{16} \mathrm{~A}$ few individual vacancies exist in the film which could be attributed to incomplete reaction or to the formation of individual heterotactic nodes. Reducing the amount of deposited molecules by applying a lower concentration of the monomer solution $\left(0.6 \times 10^{-3} \mathrm{M}\right)$ induces morphological changes in the film, from a relatively compact monolayer to fragmented domains (Fig. 4b). When an even lower concentration of precursor solution was used $\left(0.3 \times 10^{-3}\right.$ $\mathrm{M})$, branched domains with a high perimeter-to-area ratio started to appear, containing a diversity of internal structures (Fig. 4c). The individual fragments are triangular in shape and correspond to ST patterns of various order. The largest triangles can be assigned to defect-free ST-3 (Fig. 3c). Due to the fact that only the benzene rings are visible with STM, the smaller ST- $n$ ( $n$ < 3) structures are hardly resolved and simply appear as triangular-shaped network structures (Fig. 4d).

Importantly, the surface density of ST- $n$ patterns decreases rapidly with increasing $n$ order. ${ }^{39}$ Although the ST phase is favored in the low-coverage regime on a surface, increasing the coverage drives the formation of a higher-density phase, i.e. the honeycomb-like polymorph.

In brief, we presented the formation of COF from the lowsymmetry 1,3-BDBA precursor. The lamellar structure corresponds to a dense 2D honeycomb-like network, arranged in staggered stacking. The presence of homo- and heterotactic coupling modes leads to a reduced crystallinity. We could 
identify the corresponding polymorphs by STM, honeycomb networks or Sierpiński triangles, grown by on-surface synthesis on a HOPG substrate. We demonstrate that the polymorphism is controlled by varying the surface coverage. Sierpiński triangles are favored in the low coverage regime up to the third generation (ST-3).

Our results suggest that the confinement of reactions on a crystalline substrate may be an efficient way to select the desired polymorph. In future investigations host-guest chemistry for ST ${ }^{41,44-46}$ and the elucidation of the electronic properties ${ }^{26,} 27$ in such fractal confinements may reveal intriguing phenomena.

\section{Acknowledgements}

This work was supported by NSERC-Discovery Grants (D.F.P. and F.R.) and US Army Office for Scientific Research (grant \# W911NF-17-1-0126). F.R. is grateful to the Canada Research Chairs program for partial salary support and funding, Y.F. acknowledges a post-doctoral fellowship from NSERC.

\section{Conflicts of interest}

There are no conflicts to declare.

\section{References}

A. P. Cote, A. I. Benin, N. W. Ockwig, M. O'Keeffe, A. J. Matzger and O. M. Yaghi, Science, 2005, 310, 1166-1170

X. Feng X. Ding and D. Jiang Chemical Society Reviews, 2012, 41,60106022

3. P. J. Waller, F. Gándara and O. M. Yaghi, Accounts of Chemical Research, 2015, 48, 3053-3063.

C. R. DeBlase and W. R. Dichtel, Macromolecules, 2016, 49, 5297-5305. S.-Y. Ding and W. Wang, Chemical Society Reviews, 2013, 42, 548-568. P. Kuhn, M. Antonietti and A. Thomas, Angewandte Chemie International Edition, 2008, 47, 3450-3453.

G. H. V. Bertrand, V. K. Michaelis, T. C. Ong, R. G. Griffin and M. Dinca, Proceedings of the National Academy of Sciences of the United States of America, 2013, 110, 4923-4928.

X. R. Wang X. Han, J. Zhang X. W. Wu, Y. Liu and Y. Cui, J. Am. Chem. Soc. 2016, 138, 12332-12335.

9. C. Qian, Q. Y. Qi, G. F. Jiang, F. Z. Cui, Y. Tian and X. Zhao, J. Am. Chem. Soc. 2017, 139, 6736-6743.

10. Y. Jin, Y. Hu and W. Zhang, Nature Reviews Chemistry, 2017, 1, 0056.

11. F.-P. Huang, J.-L. Tian, G.-J. Chen, D.-D. Li, W. Gu, X. Liu, S.-P. Yan, D.-Z. Liao and P. Cheng, CrystEngComm, 2010, 12, 1269-1279. M. Du, C. P. Li, C. S. Liu and S. M. Fang, Coordination Chemistry Reviews, 2013, 257, 1282-1305.

T. Wang and J. F. Zhu, Surface Science Reports, 2019, 74, 97-140.

S. Clair and D. G. de Oteyza, Chem. Rev., 2019, 119, 4717-4776.

C.-Z. Guan, D. Wang and L.-J. Wan, Chemical Communications, 2012, 48 2943-2945.

16. J. F. Dienstmaier, A. M. Gigler, A. J. Goetz, P. Knochel, T. Bein, A. Lyapin, S. Reichlmaier, W. M. Heckl and M. Lackinger, ACS Nano, 2011, 5, 9737-9745. J. F. Dienstmaier, D. D. Medina, M. Dogru, P. Knochel, T. Bein, W. M. Heck and M. Lackinger, ACS Nano, 2012, 6, 7234-7242.

18. N. A. Zwaneveld, R. Pawlak, M. Abel, D. Catalin, D. Gigmes, D. Bertin and L. Porte, J. Am. Chem. Soc., 2008, 130, 6678-6679.

19. S. Clair, M. Abel and L. Porte, Chemical Communications, 2014, 50, 9627 9635.

20. S. Spitzer, A. Rastgoo-Lahrood, K. Macknapp, V. Ritter, S. Sotier, W. M. Heck and M. Lackinger, Chemical Communications, 2017, 53, 5147-5150.

21. S. Clair, F. Dumur and D. Gigmes, in Encyclopedia of Interfacial Chemistry: Surface Science and Electrochemistry, ed. K. Wandelt, Elsevier, Oxford, 2018, vol. Reference Module in Chemistry; Molecular Sciences and Chemical Engineering, pp. 424-435.
L. R. Xu, X. Zhou, W. Q. Tian, T. Gao, Y. F. Zhang, S. B. Lei and Z. F. Liu, Angew. Chem.-Int. Edit., 2014, 53, 9564-9568.

S. Kandambeth, A. Mallick, B. Lukose, M. V. Mane, T. Heine and R. Banerjee, J. Am. Chem. Soc., 2012, 134, 19524-19527.

C. Lu, Y. Li, L.-M. Wang, H.-J. Yan, L. Chen and D. Wang, Chemical communications, 2019, 55, 1326-1329.

J. Y. Yue, Y. P. Mo, S. Y. Li, W. L. Dong, T. Chen and D. Wang, Chemical Science, 2017, 8, 2169-2174.

S. Kempkes, M. Slot, S. Freeney, S. Zevenhuizen, D. Vanmaekelbergh, I. Swart and C. M. Smith, Nature physics, 2019, 15, 127.

H. Wang, X. Zhang, Z. Jiang, Y. Wang and S. Hou, Physical Review B, 2018, 97, 115451.

D. Nieckarz and P. Szabelski, Chemical Communications, 2014, 50, 68436845 .

J. Shang, Y. Wang, M. Chen, J. Dai, X. Zhou, J. Kuttner, G. Hilt, X. Shao, J. M. Gottfried and K. Wu, Nature chemistry, 2015, 7, 389.

A. Rastgoo-Lahrood, N. Martsinovich, M. Lischka, J. Eichhorn, P. Szabelski, D. Nieckarz, T. Strunskus, K. Das, M. Schmittel and W. M. Heckl, ACS Nano, 2016, 10, 10901-10911.

Y. Wang, N. Xue, R. Li, T. Wu, N. Li, S. Hou and Y. Wang, ChemPhysChem, 2019, 20, 2262-2270

X. Zhang, N. Li, G.-C. Gu, H. Wang, D. Nieckarz, P. Szabelski, Y. He, Y. Wang, C. Xie and Z.-Y. Shen, ACS Nano, 2015, 9, 11909-11915.

Q. Sun, L. Cai, H. Ma, C. Yuan and W. Xu, Chemical Communications, 2015 51, 14164-14166.

X. Zhang, R. Li, N. Li, G. Gu, Y. Zhang, S. Hou and Y. Wang, Chinese Chemical Letters, 2018, 29, 967-969.

N. Li, X. Zhang, G.-C. Gu, H. Wang, D. Nieckarz, P. Szabelski, Y. He, Y. Wang, J.-T. Lü and H. Tang, Chinese Chemical Letters, 2015, 26, 1198-1202.

X. Zhang, N. Li, L. Liu, G. Gu, C. Li, H. Tang, L. Peng, S. Hou and Y. Wang, Chemical Communications, 2016, 52, 10578-10581.

X. Zhang, G. Gu, N. Li, H. Wang, H. Tang, Y. Zhang, S. Hou and Y. Wang, RSC Advances, 2018, 8, 1852-1856.

G. Gu, N. Li, L. Liu, X. Zhang, Q. Wu, D. Nieckarz, P. Szabelski, L. Peng, B. K. Teo and S. Hou, RSC Advances, 2016, 6, 66548-66552.

Y. Mo, T. Chen, J. Dai, K. Wu and D. Wang, J. Am. Chem. Soc., 2019, 141 11378-11382.

A. Adamczyk-Woźniak, M. K. Cyrański, K. Durka, J. T. Gozdalik, P. Klimentowska, R. Rusiecki, A. Sporzyński and D. Zarzeczańska, Crystals, 2019, 9, 109.

D. Cui, J. MacLeod, M. Ebrahimi and F. Rosei, CrystEngComm, 2017, 19, 4927-4932.

Y.-P. Mo, X.-H. Liu and D. Wang, ACS Nano, 2017, 11, 11694-11700.

L. Cai, Q. Sun, M. Bao, H. Ma, C. Yuan and W. Xu, ACS Nano, 2017, 11, $3727-$ 3732.

D. Cui, J. M. MacLeod and F. Rosei, Chemical Communications, 2018, 54, 10527-10539.

D. Cui, J. M. MacLeod, M. Ebrahimi, D. F. Perepichka and F. Rosei, Chemica Communications, 2015, 51, 16510-16513.

Plas, O. Ivasenko, N. Martsinovich, M. Lackinger and S. De Feyter, Chemical Communications, 2016, 52, 68-71. 